\title{
Factors Associated with Compliance with the Treatment Protocol and Mortality in Adults with Hemophilia
}

This article was published in the following Dove Press journal: Patient Preference and Adherence

\author{
Beatriz Mac Dowell Soares (iD) 1,2 \\ Luiz Alberto Simeoni (iD) ${ }^{2}$ \\ Karlo Jozefo Quadros de Almeida (iD' \\ Jaqueline Lima de Souza' \\ Aline Mizusaki Imoto,3 \\ Melina Swain Braverman (iD ${ }^{3}$ \\ Lucas Barbosa Bezerra (ID) \\ Alexandre Jorge Teixeira Ribeiro (D) 1,3 \\ Ana Maria Costa (iD) \\ Fábio Ferreira Amorim (iD) ${ }^{1,2}$ \\ 'School of Medicine, Higher Education \\ School of Health Sciences (ESCS), \\ Faculdade de Ciências de Saúde Campos \\ Univ, Brasília, Federal District, Brazil; \\ ${ }^{2}$ Faculty of Health Sciences, University of \\ Brasilia (UnB) - Brasília, Faculdade de \\ Ciências de Saúde Campos Univ, Brasília, \\ Federal District, Brazil; ${ }^{3}$ Brasília \\ Hemocentro Foundation (FHB), SMHN \\ Conjunto A Bloco 3, Brasília, Federal \\ District, Brazil
}

Objective: Hemophilia is associated with a high prevalence of disabilities and mortality. This finding can be influenced by patient compliance with the treatment protocol. This study aims to identify compliance with a treatment protocol in adult patients with hemophilia and to evaluate the factors associated with and the impact on mortality of noncompliance with a hemophilia treatment protocol.

Methods: This was a cross-sectional study that was performed between June 2015 and May 2016, followed by a cohort to evaluate mortality until July 2019 that included all adult patients with hemophilia registered in the Federal District, Brazil.

Results: Among 138 patients enrolled in the study, 35 patients were compliant with all items of the treatment protocol (25.4\%). Regarding each item, compliance with the medical consultations was $71.0 \%(98 / 138)$; the clotting factor regimen was $65.9 \%(91 / 138)$; and the serological tests were $51.4 \%(71 / 138)$. The mortality was $7.2 \%(10 / 138)$. Noncompliance with any aspect of the protocol was associated with mortality: medical consultations $(\mathrm{p}<0.001)$, clotting factor regimen $(\mathrm{p}=0.013)$, and serological tests $(\mathrm{p}=0.006)$. All deaths occurred in those who did not comply with the protocol, and the majority were due to bleeding. Patients who were noncompliant with all protocol items showed the highest mortality $(50.0 \%, 5 / 10)$. Treatment at the hemophilia treatment center (OR: $2.388 ; 95 \%$ CI: $1.052-5.418, \mathrm{p}=0.037$ ) was positively and independently associated with compliance with the protocol in multivariate analysis.

Conclusion: Noncompliance with the treatment protocol was high. Treatment at a hemophilia treatment center was positively and independently associated with compliance with the protocol, which reinforces the importance of comprehensive care by a multidisciplinary team.

Keywords: hemophilia A, hemophilia B, patient compliance, medication adherence, treatment adherence and compliance, clinical protocols

\section{Introduction}

Hemophilia is an inherited X-linked bleeding disorder characterized by factor VIII (hemophilia A) or factor IX deficiency (hemophilia B). The goal of hemophilia treatment is to reduce bleeding episodes to prevent squeal due to hemorrhagic events and mortality risk, and treatment is mainly based on the episodic and/or prophylactic replacement of clotting factor concentrates. ${ }^{1-3}$ Despite an increase in treatment advances in recent years, hemophilia remains associated with a high prevalence of disabilities and mortality in many countries. ${ }^{2,4,5}$ One of the factors
Correspores

School of Medicine, Escola Superior de Ciências da Saúde (ESCS), SMHN

Conjunto A Bloco 0I Edifício Fepecs -

Asa Norte, Brasília - DF, Brasília CEP:

707I0-907, Federal District, Brazil,

Tel +55 61999836446

Email biamacdowell@uol.com.br
Patient Preference and Adherence 2020:14 2279-2285

2279

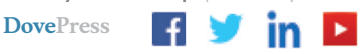

http://doi.org/10.2147/PPA.S279401 
that may influence this finding is compliance with the hemophilia treatment protocol, which may be due to problems related either to the patient or healthcare professional adherence to the protocol recommendations. In addition to other chronic diseases, such as diabetes mellitus and systemic arterial hypertension, a challenge for healthcare professionals is ensuring that patients comply with their treatments. It is known that most chronic diseases require multidisciplinary team care. ${ }^{6-9}$ A recent study carried out in the United States showed that nonattendance at scheduled visits to a hemophilia treatment center (HTC) was associated with increased visits to the emergency department and hospitalizations. ${ }^{10}$

Brazil has the world's fourth-largest population of hemophilic patients (10,395 patients with hemophilia A and 2037 with hemophilia B), according to the Annual Global Survey 2017 of the World Federation of Hemophilia, after the United States, India, and China. ${ }^{11}$ In Brazil, the Unified Health System (SUS) is responsible for providing free universal public coverage of healthcare services for the entire population. $^{12}$ Regarding hemophilia care, the Brazilian Ministry of Health has implemented public policies to improve treatment, especially by making higher investments mainly in the acquisition and the regular free-of-charge distribution of plasma-derived or recombinant coagulation factors, aiming to reduce the risks of complications. ${ }^{4,13-16}$

Thus, this study aimed (i) to identify compliance with a treatment protocol among adult patients with hemophilia and (ii) to evaluate the factors associated with and impact on mortality of noncompliance with a hemophilia treatment protocol.

\section{Methods}

\section{Patients}

This study was a cross-sectional study performed between June 2015 and May 2016, followed by a cohort to evaluate mortality until July 2019. All patients aged $>18$ years with hemophilia A or B registered since 2012 in the Brazilian Unified Health System in the Federal District, Brazil, according to the Brazilian registry of people with hereditary coagulopathies (National Hemovida System of Coagulopathies), was included. ${ }^{16}$

The Ethics Committee of the Education and Research Foundation of Health Sciences (FEPECS), Brasília, Federal District, Brazil approved the study that was conducted following the Declaration of Helsinki. Since the study was observational, without any specific intervention, using only anonymized medical records' data and other institutional clinical information that generated results in an aggregate manner, and does not allow the identification of research participants, the written consent was not necessary according to the Resolution of the Brazilian National Research Ethics Council.

\section{Data Collection}

Patient age, type of hemophilia (A or B), severity of hemophilia (mild, moderate or severe), treatment at an HTC, medical consultations per year, type of treatment (prophylactic or ondemand), dose and type of clotting factor (recombinant or plasma-derived coagulation factor), inhibitor antibody tests, and serological tests for infectious diseases (Chagas disease antibody, total hepatitis B core antibody - anti-HBc, hepatitis B surface antigen - HbsAg, hepatitis B surface antibody - antiHBs, anti-hepatitis $\mathrm{C}$ virus antibody - anti-HCV, anti-human T-cell lymphotropic virus types 1 and 2 antibody - anti-HTLV$1 / 2$, and anti-human immunodeficiency antibody - anti-HIV) were collected from medical records. All serological tests for infectious diseases were performed by the chemiluminescence method $\left(\mathrm{Abbott}^{\mathbb{R}}\right)$, which were processed at the Brasília Hemocentro Foundation as part of the hemophilia treatment protocol.

Hemophilia severity was defined based on the lowest factor VIII or IX plasma residual activity: severe hemophilia when clotting factor levels were below $1 \%$, moderate hemophilia when levels were between $1 \%$ and $5 \%$, and mild hemophilia when levels were above $5 \%$ up to $40 \%{ }^{3}$

The Brasília Hemocentro Foundation (BHF) is the HTC for the treatment of inherited coagulation disorders in the Federal District, Brazil. In the last eight years, the BHF implemented several improvements in the healthcare of patients with hemophilia, such as the implementation of the treatment protocol that promotes comprehensive care by a multidisciplinary team with medical, psychological, and physical therapy consultations, a hemostasis laboratory, and pharmaceutical support with home delivery. This protocol includes recommendations for frequency of multidisciplinary team consultations (at least 1 per year for mild hemophilia and 2 per year for moderate/severe hemophilia), type of treatment (prophylaxis or on-demand according to hemophilia severity), type and doses of clotting factor prescription (recombinant factor VIII is indicated for patients until 30 years old and plasma-derived factor for patients above 30 years old), and a laboratory test schedule.

In this study, compliance with the hemophilia treatment protocol was defined through the following checklist: 
Table I Baseline Data of Adult Patients with Hemophilia $(\mathrm{n}=$ 138)

\begin{tabular}{|l|l|}
\hline Age, years, mean (SD) & $\begin{array}{l}36.4 \\
\mathbf{( 1 2 . 5 )}\end{array}$ \\
\hline Hemophilia A, $\mathrm{n}(\%)$ & $104(75.4)$ \\
\hline $\begin{array}{l}\text { Hemophilia severity, } \mathrm{n}(\%) \\
\text { Mild }\end{array}$ & $24(17.4)$ \\
Moderate & $8(5.8)$ \\
Severe & $106(76.8)$ \\
\hline $\begin{array}{l}\text { Compliance to the hemophilia treatment protocol, } \\
\mathrm{n} \text { (\%) }\end{array}$ & $35(25.4)$ \\
Medical consultations & $98(71.0)$ \\
Clotting factor regimen & 9 I (65.9) \\
Serological tests & $7 \mathrm{I}(51.4)$ \\
\hline Mortality, $\mathrm{n}$ (\%) & $10(7.2)$ \\
Hemorrhage, $\mathrm{n}$ (\% of deaths) & $6(60.0)$ \\
Liver cancer, $\mathrm{n}$ (\% of deaths) & $\mathrm{I}(10.0)$ \\
Lymphoma, $\mathrm{n}$ (\% of deaths) & $\mathrm{I}(10.0)$ \\
Sepsis, $\mathrm{n}$ (\% of deaths) & $\mathrm{I}(10.0)$ \\
Unknown, $\mathrm{n}$ (\% of deaths) & $\mathrm{I}(10.0)$ \\
\hline
\end{tabular}

Abbreviation: SD, standard deviation.

1. Medical consultations: number of medical consultations in agreement with the recommended amount over the previous year (at least once a year for patient with mild haemophilia, and at least every 6 months for moderate and severe haemophilia)

2. Clotting factor regime: type of treatment (prophylactic or on-demand), dose of clotting factor (UI/Kg and weekly frequency of coagulation factor injections), and type of clotting factor (recombinant or plasma-derived coagulation factor) in agreement with the protocol recommendations.

3. Serological tests: number of tests for infectious diseases and inhibitor antibodies according to the recommended number over the previous year (at least once a year)

Failure to comply with any of the items was considered noncompliant with the protocol treatment.

\section{Statistical Analysis}

Quantitative data are expressed as the mean \pm standard deviation (SD) or as the median and interquartile range (25-75th percentile) according to their distribution assessed by the Kolmogorov-Smirnov test. Categorical variables are expressed as numbers and percentages (\%). Student's t-test or the Mann-Whitney test was used to compare quantitative variables, as appropriate. Contingency tables were used for categorical variables, and Pearson's chi-square test $(\chi 2)$ or Fisher's exact test was used, as appropriate. To evaluate independent factors associated with noncompliance with the protocol, noncollinear variables associated with noncompliance with the protocol with a p-value $<0.20$ in the univariate analysis were assessed using backward stepwise logistic regression analysis. Statistical analyses were performed using IBM Statistical Package for Social Sciences 20.0 for Mac (SPSS 20.0 Mac, SPSS Inc., Chicago, Illinois, USA). The level of statistical significance was defined as a twosided $\mathrm{P}$-value $<0.05$.

\section{Results}

The study included 138 adult patients with hemophilia, mainly hemophilia A $(75.4 \%, 104 / 138)$. Table 1 shows the baseline data. The mean age of enrolment in the study was $36.4 \pm 12.5$ years. Severe hemophilia was the most common $(76.8 \%, 106 / 138)$, and 35 patients were compliant with all items of the treatment protocol $(25.4 \%)$. Regarding each aspect of the protocol individually, compliance with medical consultations, clotting factor regimen, and serological tests were $71.0 \%$ (98/138), $65.9 \%$ (91/138), and 51.4\% (71/138), respectively. The mortality rate during the study period was $7.2 \%(10 /$ 138). Regarding the causes of death, $60 \%$ occurred due to spontaneous hemorrhage $(6 / 10), 10 \%$ due to liver cancer $(1 / 10), 10 \%$ due to lymphoma $(1 / 10)$, and $10 \%$ due to sepsis $(1 / 10)$. The cause of death was unknown in one patient $(10 \%, 1 / 10)$.

Table 2 compares compliant and noncompliant patients to the protocol. In the univariate analysis, a statistically significant association was observed between compliance with the protocol and treatment at the HTC $(p=0.018)$. No other statistically significant differences were found when comparing the groups regarding age, severity or type of hemophilia, presence of inhibitors, and serologies for infectious diseases. Using multivariate analysis, treatment in the HTC $(p=0.037)$ remained associated with compliance with the protocol.

Table 3 shows that treatment in the HTC (OR: 2.388; 95\% CI: 1.052-5.418) resulted in a positive and independent association with compliance with the protocol in multivariate analysis.

Table 4 shows that the number of items in compliance with the treatment protocol was associated with mortality $(p<0.001)$. All deaths occurred in patients who were 
Table 2 Analysis (Univariate and Multivariate) of the Factors Associated with Compliance with the Treatment Protocol in Adult Patients with Hemophilia

\begin{tabular}{|c|c|c|c|c|}
\hline & $\begin{array}{l}\text { Compliance to the } \\
\text { Protocol } \\
(n=35)\end{array}$ & $\begin{array}{l}\text { Noncompliance to the Protocol } \\
(n=103)\end{array}$ & $\begin{array}{l}\text { P-value } \\
\text { Univariate } \\
\text { Analysis }\end{array}$ & $\begin{array}{l}\text { P-value } \\
\text { Multivariate } \\
\text { Analysis }\end{array}$ \\
\hline Age, years, mean (SD) & $37.7(14.6)$ & $36.0(11.8)$ & 0.612 & - \\
\hline Non-treatment at HCT, n (\%) & $13(37.1)$ & $62(60.2)$ & 0.018 & 0.039 \\
\hline Hemophilia A, n (\%) & $29(82.9)$ & $75(72.8)$ & 0.234 & - \\
\hline Severe hemophilia, n (\%) & $27(77.1)$ & $77(74.8)$ & 0.777 & - \\
\hline Positive Chagas disease antibody, n (\%) ${ }^{a}$ & $3(8.6)$ & $2(2.0)$ & 0.109 & 0.088 \\
\hline Positive anti-HCV, n (\%) ${ }^{\mathrm{b}}$ & $13(37.0)$ & $51(50.5)$ & 0.173 & 0.309 \\
\hline Positive anti-HTLV-I/2, n (\%) ${ }^{\mathrm{b}}$ & $0(0.0)$ & $2(2.0)$ & 0.550 & - \\
\hline Positive anti-HBc, n (\%) ${ }^{b}$ & $5(14.3)$ & $22(14.3)$ & 0.338 & - \\
\hline Positive HBsAg, n (\%) ${ }^{\mathrm{b}}$ & $0(0.0)$ & $2(2.0)$ & 0.402 & - \\
\hline Positive anti-HBc + positive $\mathrm{HBsAg}, \mathrm{n}(\%)^{\mathrm{b}}$ & $0(0.0)$ & $2(2.0)$ & 0.402 & - \\
\hline Positive anti-HIV, n (\%) & I (2.9) & $5(5.0)$ & 0.509 & - \\
\hline Positive inhibitor antibodies, $\mathrm{n}(\%)^{c}$ & $2(5.7)$ & $4(4.3)$ & 0.726 & - \\
\hline
\end{tabular}

Notes: ${ }^{a} 3$ patients did not undergo Chagas disease antibody and anti-HIV serology. ${ }^{b} 2$ patients did not undergo anti-HBc, $\mathrm{HBsAg}$, anti-HCV, and anti-HTLV I/2 serologies. ${ }^{\mathrm{c}} 9$ patients did not undergo the measurement of inhibitor antibodies.

Abbreviations: HTC, hemophilia treatment center; Anti-HBc, total hepatitis B core antibody; anti-HCV, anti-hepatitis C virus antibodies; anti-HTLV-I and HTLV-2, antihuman T-cell lymphotropic virus types I and 2 antibodies; anti-HIV, anti-human immunodeficiency antibodies; SD, standard deviation.

Table 3 Final Multivariate Analysis of Factors Associated with Compliance of the Treatment Protocol in Adult Patients with Hemophilia

\begin{tabular}{|l|l|}
\hline & OR (95\% CI) \\
\hline Treatment at HCT & $2.388(\mathrm{I} .052-5.418)$ \\
Positive anti-HCV & $0.657(0.293-1.476)$ \\
Positive Chagas disease antibody & $0.194(0.029-1.275)$ \\
\hline
\end{tabular}

Notes: Hosmer-Lemeshow test: $\chi^{2}=1.220 ; \mathrm{df}=2 ; \mathrm{p}$-value $=0.543$.

Abbreviations: HTC, hemophilia treatment center; anti-HCV, anti-hepatitis C virus antibodies; OR, odds ratio; $\mathrm{Cl}$, confidence interval.

noncompliant with the protocol. Noncompliance with any aspect of the protocol was associated with mortality: medical consultations $(\mathrm{p}<0.001)$, clotting factor regimen $(\mathrm{p}=0.013)$, and serological tests $(\mathrm{p}=0.006)$. The group that did not comply with all items of the protocol showed the highest mortality $(50.0 \%, 5 / 10)$.

\section{Discussion}

This study found that noncompliance with the treatment protocol remains high in adults with hemophilia, even in a country that has been implementing public health policies to improve the treatment and provides public healthcare assistance and free-of-charge distribution of coagulation factors to hemophilic patients, such as Brazil. ${ }^{4,12,15,17}$ In other studies, the level of compliance ranged from $43 \%$ to $87 \%$, but they evaluated different aspects of hemophilia treatment, mainly adherence to prophylactic treatment, and included children and adolescents. ${ }^{17-21}$ The present study showed a much lower rate of compliance, but it evaluated other aspects of the treatment protocol in addition to the adherence to clotting factor treatment prescribed and included only adults that are associated with a higher chance of noncompliance than younger age patients. ${ }^{17-}$ 19,22 Since our study only included adult patients, this aspect may be related to the no association observed between age and compliance in the present study.

Although the compliance with all protocol items in the present study was only $25.4 \%$, there was great variation between the different aspects evaluated, from $51.4 \%$ for serological tests to $71.0 \%$ for medical consultations. The compliance with the clotting factor regimen was $65.9 \%$. A Dutch study evaluating the adherence to coagulation factor doses prescribed showed $43 \%$ adherence in patients' selfadministration, $63 \%$ adherence in parents administration, $37 \%$ suboptimal adherence in patients, and $29 \%$ adherence in parents administration. ${ }^{19}$ Another study showed higher adherence to coagulation factors prescribed from $80 \%$ to $87 \%$ in six European countries. ${ }^{18}$ In the United Kingdom, a study evaluating only adolescents and young adults with severe hemophilia showed high compliance, with only $18 \%$ that self-reported missing prophylaxis doses. ${ }^{17}$ Regarding medical consultations, a study showed that $12 \%$ of patients did not attend scheduled appointments at a United States HTC. ${ }^{10}$ In general, when comparing the 
Table 4 Compliance with the Protocol and Mortality in Adult Patients with Hemophilia

\begin{tabular}{|c|c|c|c|}
\hline & $\begin{array}{l}\text { Survival } \\
(n=\mid 28)\end{array}$ & $\begin{array}{l}\text { Non-Survival } \\
(n=10)\end{array}$ & P-value \\
\hline Compliance with medical consultations, $\mathrm{n}(\%)$ & $97(75.8)$ & $I(10.0)$ & $<0.001$ \\
\hline Compliance with clotting factor regimen, $\mathrm{n}(\%)$ & $88(68.8)$ & $3(30.0)$ & 0.013 \\
\hline Compliance with serological tests, $\mathrm{n}(\%)$ & $70(54.7)$ & $I(10.0)$ & 0.006 \\
\hline Number of items in compliance with the protocol, $\mathrm{n}(\%)$ & $35(27.3)$ & $0(0.0)$ & $<0.001$ \\
\hline 3 & & & \\
\hline 2 & $63(49.2)$ & I (I0.0) & \\
\hline I & $24(18.8)$ & $4(40.0)$ & \\
\hline 0 & $6(4.7)$ & $5(50.0)$ & \\
\hline
\end{tabular}

studies, compliance was high in countries where healthcare systems are mostly public. ${ }^{17-21,23}$ Indeed, a United States study showed that the cost was mentioned by $45 \%$ of hemophilia nurses as a major barrier to prophylaxis adherence. ${ }^{22}$ However, our study showed low compliance with the protocol even in a country that has adopted a public healthcare system with free and universal coverage for its population, including treatment with high-cost products, which shows that other factors are also important. 4,12,16,17

Protocols are essential to achieve better physical and psychosocial health and quality of life, focusing on relevant aspects, such as monitoring pharmacological treatment, bleeding, and the presence of inhibitors. Additionally, scheduled appointments as part of the protocol are opportunities for health promotion and prevention of harm. ${ }^{3,10}$ Although the high rate of nonadherence observed in our study may also be occurring in other Brazilian regions, a recent study showed a progressive decrease in the mortality of patients with hemophilia in Brazil, ${ }^{2}$ which may be related to the improvement in hemophilia care due to Brazilian healthy public policies in recent years. ${ }^{2}$ However, this result could be even better if new strategies were adopted, aiming to promote greater treatment protocol compliance, especially when all deaths occurred in patients who were noncompliant with protocol in our study.

In this article, most deaths occurred due to bleeding. Hemorrhage and its complications are still the main causes of death in hemophilia, as shown in recent studies. ${ }^{2,21,24-26}$ Regarding the association between the incidence of hemorrhagic events and noncompliance with the protocol, a study that evaluated nonattendance at appointments scheduled in the United States HTC showed there was an association between nonattendance and consultations in emergency services and hospitalizations. ${ }^{10}$ A study showed that nonadherence to prophylaxis was associated with the number of hemarthrosis in patients with hemophilia aged 12 to 25 years. ${ }^{21}$ Indeed, a study in children and adolescents from 33 HTCs in 20 countries found that regular and frequent prophylaxis decreased the risk of intracranial hemorrhage compared with infrequent or non-existent prophylaxis. ${ }^{27}$ These studies suggest that when patients are not submitted to regular clinical follow-up, they are at high risk for acute events, such as bleeding.

For the development of new policies that aim to promote improvement in compliance with the protocol, it is important to recognize the factors associated with noncompliance in hemophilic patients. In this study, noncompliance with the clinical treatment protocol was associated with nontreatment in an HTC, which may show the importance of comprehensive care by a multidisciplinary team and family support. Hemophilia is a rare disease with complex treatment. In this sense, health professionals must have the knowledge and experience to address the peculiarities of the treatment of hemorrhagic disorders. These patients need comprehensive care by a multidisciplinary team, who can promote better physical and psychosocial health, in addition to ensuring continuity of care. ${ }^{1,12,28}$ Moreover, compliance with the treatment protocol is a complex and multidimensional phenomenon that is influenced by multiple aspects, such as clinical, personal, social, and organizational factors. ${ }^{17,29,30}$ Thus, comprehensive multidisciplinary care at an HTC is essential to promote patient compliance, as demonstrated in the present study, which found greater compliance in the protocol in patients treated at an HTC. A European survey showed that the time spent at an HTC and the quality of the relationship with the hematologist and the nurse were associated with greater adherence to treatment in hemophilia. $^{18}$ Additionally, the implementation of an HTC decreased costs and improved patient outcomes in different studies, ${ }^{29,31-33}$ and patients who had undergone 
care in an HTC had a significant reduction in the risk of death. $^{29,32}$

No other factor evaluated in this study, such as age and severity or type of hemophilia, was independently associated with nonadherence to the treatment protocol. Regarding age, it is important to note that our study only evaluated adults, and previous studies reported higher compliance among children and adolescents than among adults. $^{17-21}$ As in other chronic diseases, the association between disease severity and protocol compliance remains uncertain. $^{10,34,35}$ A North American study showed no association between the severity of the disease and the failure to attend scheduled appointments, one aspect of compliance evaluated in the present study. ${ }^{10}$

There are limitations to our study. Hemophilia is a rare disease, and therefore, it is difficult to have a large cohort. This study did not measure other potential characteristics for compliance with the protocol, such as between educational level, income, health status, access to transportation, and the quality of the relationship with the patient and multidisciplinary team. In this respect, the educational level and low income of the majority of the Brazilian population are still factors to be considered concerning the understanding of hemophilia and how the healthcare system is organized in Brazil. Given this reality, in 2011, BHF started delivering coagulation factors to the home, accompanied by psychosocial assessment and guidance on the storage and application of the products by nurses, pharmaceutics, and social workers. Due to the peculiarities of treatment in adults and children, like the care given by parents to patients, we decided included only adults in the present study, so our results should only be generalized to patients aged $>18$ years. Finally, it was not possible to perform a multivariate analysis to assess the outcome of mortality, since the number during the study period was insufficient.

\section{Conclusion}

Noncompliance with the protocol was high in this group of adult patients with hemophilia. Compliance with the treatment protocol was positively and independently associated with treatment at the HTC. All deaths occurred in patients who did not comply with the protocol, and the majority were due to bleeding. Among the deaths, 50\% of the patients did not comply with any item of the protocol, and the other $50 \%$ of the deaths fulfilled only one or two items of the protocol. These results reinforce the importance of care by a multidisciplinary team, and the preventive treatment and protocol.
All members of the healthcare team should be aware of strategies to promote the continuous improvement of the treatment and patient compliance with the protocol. Preventive efforts should be focused on minimizing the occurrence of bleeding, especially with the correct application of the treatment protocol and monitoring of patients by a multidisciplinary team.

\section{Disclosure}

The authors report no conflicts of interest for this work.

\section{References}

1. Cohen AJ, Kessler CM. Treatment of inherited coagulation disorders. Am J Med. 1995;99(6):675-682. doi:10.1016/S0002-9343(99)80256-3

2. Jardim LL, van der Bom JG, Caram-Deelder C, Gouw SC, Leal Cherchiglia M, Meireles Rezende S. Mortality of patients with haemophilia in Brazil: first report. Haemophilia. 2019;725(3):e146-52.

3. Srivastava A, Brewer AK, Mauser-Bunschoten EP, et al. Guidelines for the management of hemophilia. Haemophilia. 2013;19(1):e1-47.

4. Guedes VG, Thomas S, Wachholz PA, Souza SAL. Challenges and perspectives in the treatment of patients with haemophilia in Brasil. Rev Assoc Med Bras. 2018;64(10):872-875. doi:10.1590/18069282.64.10.872

5. Plug I, Van Der Bom JG, Peters M, et al. Mortality and causes of death in patients with hemophilia, 1992-2001: a prospective cohort study. J Thromb Haemost. 2006;4(3):510-6. doi:10.1111/j.15387836.2006.01808.x

6. American Diabetes Association. Standards of medical care in diabetes-2014. Diabetes Care. 2014;37(Suppl 1):S14-80. doi:10.2337/ de14-S014

7. Bodenheimer T, Lorig K, Holman H, Grumbach K. Patient selfmanagement of chronic disease in primary care. JAMA. 2002;288 (19):2469-2475. doi:10.1001/jama.288.19.2469

8. Coleman MT, Newton KS. Supporting self-management in patients with chronic illness. Am Fam Physician. 2005;72(8):1503-1510.

9. Wagner EH. The role of patient care teams in chronic disease management. BMJ. 2000;320(7234):569-572. doi:10.1136/bmj.320.7234.569

10. Barry V, Steffens C, Mattis S, Sidonio RF Jr, Tran DQ, Kempton CL. A cross-sectional study of non-attendance among patients at a US hemophilia treatment center 2010-2014. Haemophilia. 2018;24 (6):902-910. doi:10.1111/hae.13553

11. World Federation of Hemophilia. Annual Global Survey 2017. Montreal, Canada: World Federation of Hemophilia; 2017.

12. Massuda A, Hone T, Leles FAG, de Castro MC, Atun R. The Brazilian health system at crossroads: progress, crisis and resilience. BMJ Glob Health. 2018;3(4):e000829. doi:10.1136/bmjgh-2018000829

13. Carmo RA, Martins ML, Chaves DG, Dezanet LNC. Prevalence and risk factors associated with hepatitis $\mathrm{C}$ among Brazilian male patients with haemophilia: A long-term follow-up. Haemophilia. 2019;25 (3):447-455. doi:10.1111/hae.13728

14. Ferreira AA, Leite IC, Bustamante-Teixeira MT, Guerra MR. Hemophilia A in Brazil - epidemiology and treatment developments. J Blood Med. 2014;5:175-184. doi:10.2147/JBM.S68234

15. Rezende SM, Pinheiro K, Caram C, Genovez G, Barca D. Registry of inherited coagulopathies in Brazil: first report. Haemophilia. 2009;15 (1):142-9. doi:10.1111/j.1365-2516.2008.01907.x

16. Rezende SM, Rodrigues S, Brito K, et al. Evaluation of a web-based registry of inherited bleeding disorders: a descriptive study of the Brazilian experience with HEMOVIDAweb Coagulopatias. Orphanet J Rare Dis. 2017;12(1):27. doi:10.1186/s13023-016-0560-6 
17. Van Os SB, Troop NA, Sullivan KR, Hart DP. Adherence to prophylaxis in adolescents and young adults with severe haemophilia: a quantitative study with patients. PLoS One. 2017;12(1):e0169880. doi:10.1371/journal.pone.0169880

18. De Moerloose P, Urbancik W, Van Den Berg HM, et al. A survey of adherence to haemophilia therapy in six European countries: results and recommendations. Haemophilia. 2008;14(5):931-938. doi:10.1111/ j.1365-2516.2008.01843.x

19. Schrijvers LH, Beijlevelt-van der Zande M, Peters M, et al. Adherence to prophylaxis and bleeding outcome in haemophilia: a multicentre study. $\mathrm{Br}$ J Haematol. 2016;174(3):454-60. doi:10.1111/bjh.14072

20. Lee Mortensen G, Strand AM, Almén L. Adherence to prophylactic haemophilic treatment in young patients transitioning to adult care: A qualitative review. Haemophilia. 2018;24(6):862-872. doi:10.1111/ hae. 13621

21. Pérez-Robles T, Romero-Garrido JA, Rodriguez-Merchan EC, Herrero-Ambrosio A. Objective quantification of adherence to prophylaxis in haemophilia patients aged 12 to 25 years and its potential association with bleeding episodes. Thromb Res. 2016;143:22-27. doi:10.1016/j.thromres.2016.04.015

22. Geraghty S, Dunkley T, Harrington C, Lindvall K, Maahs J, Sek J. Practice patterns in haemophilia A therapy - global progress towards optimal care. Haemophilia. 2006;12(1):75-81. doi:10.1111/j.13652516.2006.01189.x

23. Hacker MR, Geraghty S, Manco-Johnson M. Barriers to compliance with prophylaxis therapy in haemophilia. Haemophilia. 2001;7(4):392396.

24. Chang CY, Yeh GC, Lin SY, Tseng IJ, Tsai CH, Lee YW. Trends in the epidemiology, diagnosed age and mortality rate of haemophiliacs in Taiwan: a population-based study, 1997-2009. Haemophilia. 2014;20(4):535-40. doi:10.1111/hae.12373

25. Darby SC, Kan SW, Spooner RJ, et al. Mortality rates, life expectancy, and causes of death in people with hemophilia A or B in the United Kingdom who were not infected with HIV. Blood. 2007;110 (3):815-825. doi:10.1182/blood-2006-10-050435
26. Reitter S, Waldhoer T, Vutuc C, Lechner K, Pabinger I. Survival in a cohort of patients with haemophilia at the haemophilia care center in Vienna, Austria, from 1983 to 2006. Haemophilia. 2009;15(4):88893. doi:10.1111/j.1365-2516.2009.02029.x

27. Andersson NG, Auerswald G, Barnes C, et al. Intracranial haemorrhage in children and adolescents with severe haemophilia A or Bthe impact of prophylactic treatment. $B r J$ Haematol. 2017;179 (2):298-307. doi:10.1111/bjh.14844

28. Aledort LM, Coates J. Can managed care support hemophilia in the $21 \mathrm{st}$ century? Am J Hematol. 2005;79(2):171-173. doi:10.1002/ajh.20409

29. Dalton DR. Hemophilia in the managed care setting. Am J Manag Care. 2015;21(6 Suppl):S123-30.

30. Lamiani G, Strada I, Mancuso ME, et al. Factors influencing illness representations and perceived adherence in haemophilic patients: a pilot study. Haemophilia. 2015;21(5):598-604. doi:10.1111/hae.12654

31. Larsen NM, Oakley MA, Soucie M. Mortality rate and risk factors among males with hemophilia receiving care in U.S. Hemophilia Treatment Centers (HTCs). Blood. 2007;110(11):1143. doi:10.1182/ blood.V110.11.1143.1143

32. Soucie JM, Nuss R, Evatt B, et al. Mortality among males with hemophilia: relations with source of medical care. The Hemophilia Surveillance System Project Investigators. Blood. 2000;96(2):437442.

33. Soucie J, Symons J, Evatt B, et al. Hemophilia Surveillance System Project Investigators. Home-based factor infusion therapy and hospitalization for bleeding complications among males with haemophilia. Haemophilia. 2001;7(2):198-206. doi:10.1046/j.1365-2516.200 1.00484.x

34. Catz SL, McClure JB, Jones GN, Brantley PJ. Predictors of outpatient medical appointment attendance among persons with HIV. AIDS Care. 1999;11(3):361-373. doi:10.1080/09540129947983

35. Mugavero MJ, Lin HY, Willig JH, et al. Missed visits and mortality in patients establishing initial outpatient HIV treatment. Clin Infect Dis. 2009;48(2):248-256. doi:10.1086/595705
Patient Preference and Adherence

\section{Publish your work in this journal}

Patient Preference and Adherence is an international, peer-reviewed, open access journal that focusing on the growing importance of patient preference and adherence throughout the therapeutic continuum. Patient satisfaction, acceptability, quality of life, compliance, persistence and their role in developing new therapeutic modalities and compounds to optimize clinical outcomes for existing disease states are major areas of interest for the journal. This journal has been accepted for indexing on PubMed Central. The manuscript management system is completely online and includes a very quick and fair peer-review system, which is all easy to use. Visit http:/ www.dovepress.com/testimonials.php to read real quotes from published authors. 